\title{
Dene and Western medicine meet in image-based storytelling
}

\author{
Cite as: CMAJ 2018 September 10;190:E1085-6. doi: 10.1503/cmaj.180384
}

A related article by Boivin will be published September 17, 2018.

W e crouch to make it through the door of the turboprop plane, and then sit across the aisle from each other. We shout to be heard and scream with laughter. Lisa Boivin and I are on our way to host a workshop with family physicians in Sioux Lookout, Ontario, a town that also provides health care to 33 First Nations communities, including the Nishnawbe Aski communities north of Sioux Lookout and the Treaty 3 community of Lac Seul First Nation.

The family physicians had asked me to teach about narrative medicine, and how they could incorporate story-based approaches into their practice. ${ }^{1,2}$ This request raised questions with which I have grappled: about how medicine has (and has not) opened itself to Indigenous worldviews and ways of knowing. Even narrative medicine, which offers methods of engaging with patients' stories of illness, has tended to focus on Western canonical narratives, forms and genres. ${ }^{3,4}$ I decided to invite Lisa to co-teach and introduce Indigenous methods that might provide alternative ways of seeing and knowing medicine through an Indigenous lens.

As the plane climbs into the clouds, we look down at the landscape. With the many lakes in view, Lisa speaks of her deep connection with the land. As she leaves and returns to her own community, Deninu Kue First Nation, she says her first and last views are always of the lakes below. "We are the land. The land is us," Lisa says.

Over the three days that we travel and work together, I witness Lisa sharing her personal journey through health care. Her experiences - and those of her father,

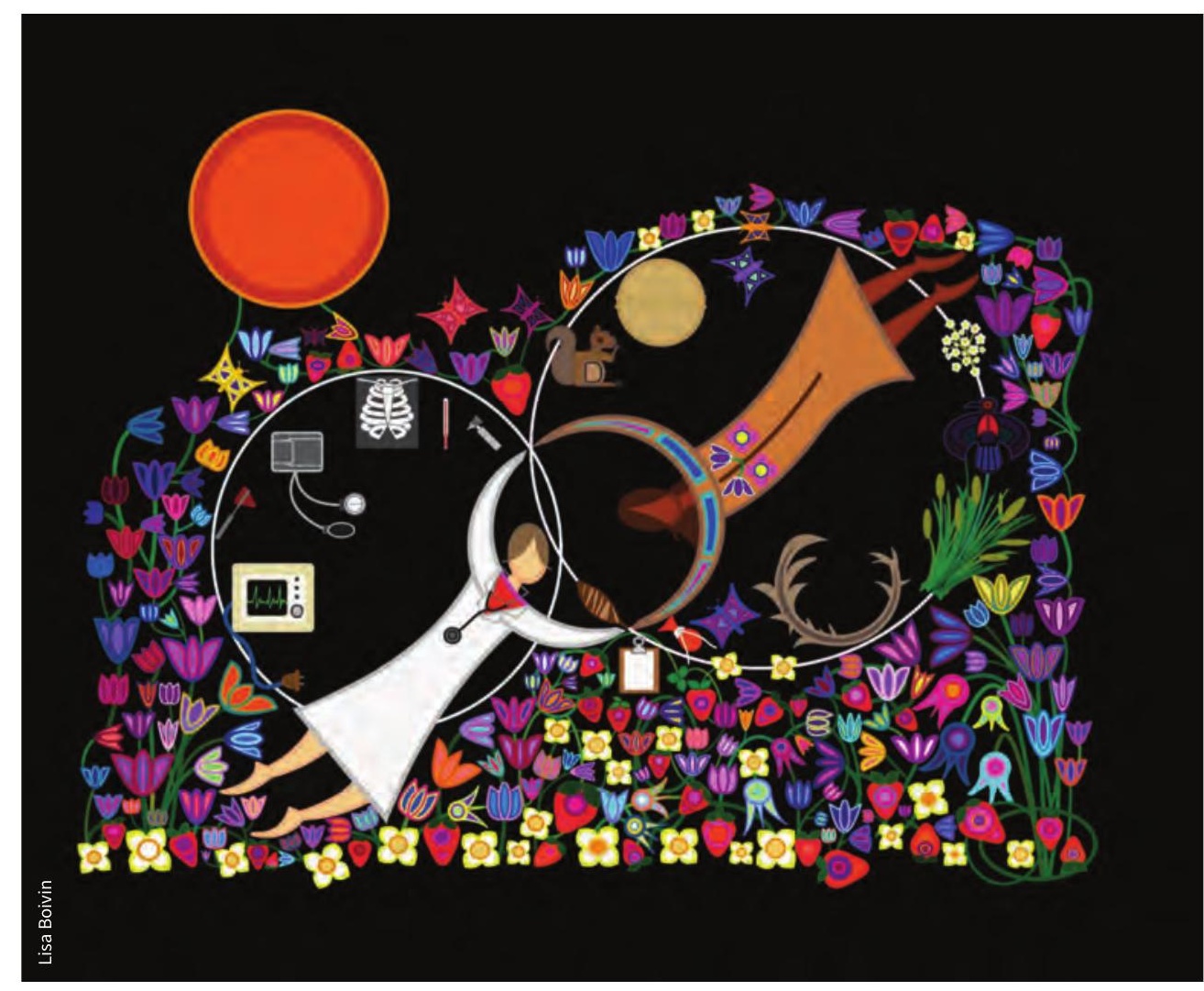

"Sharing Bioethics" shows dozens of land-based teachings. At first glance, the viewer can identify two intersecting circles of medicine. A circle surrounds a clinician in a white coat and bioscientific tools of medicine, such as a stethoscope and $x$-rays. In the Dene circle of medicine, plant medicine and Dene medicine stories are represented by a medicine bundle, strawberries and antlers. In the space where the circles intersect, the two healers also connect in a new space of relationship and possibility. In this new circle of medicine, neither knowledge or perspective is lost.

who contracted polio in childhood - are also part of the larger story of colonization and the ways settlement was enacted through health care. Relocation away from communities for health care, residential schooling, the "Sixties Scoop" of Indigenous children into the child welfare system: all affected the health and wellbeing of Indigenous communities and families, and continue to do so. ${ }^{5,6}$ Many of these dislocations also ruptured the communities' ability to be in proximity to the land, which is central to wellness and to cultural continuity.

The personal story of Lisa's family is one of inequity but also of resilience and vibrant cultural survivance. ${ }^{7}$ Lisa is a doctoral student in Rehabilitation Sciences at 
the University of Toronto; she is also learning traditional Dene medicine stories. She brings biomedicine and Dene medicine together through her image-based narratives. From her Dene knowledge and experience, "everything that has to do with ill health as understood within Western medicine is a result of separation from land and land-based practices."

Lisa, a self-taught artist, describes herself as an image-based storyteller, recounting the story of her family. She began painting while studying bioethics and rehabilitation science. The imagery she uses arose from a dual desire to find new means to communicate her knowledge and experience, and to find alternative ways to participate in the Western academy. Images have high impact and can be absorbed holistically and immediately, without recourse to the temporality of logic and reasoning. They unsettle these academic ways of knowing by presenting the familiar in new ways, alongside new knowledge, and therefore standing in new relations to Indigenous knowledges and to the viewer's own knowledge.

Lisa links her image-based work with her academic interest in bioethics. The principles of autonomy, beneficence and nonmaleficence comprise the foundation for the ethical practice of medicine. Often overlooked within this accepted framework is the history upon which consent is premised, specifically the history of Indigenous peoples in Canada. How, she questions through her work, "can consent exist when we are not recognized or treated as autonomous individuals?"

Lisa says her painting practice began "with my own inability to connect with peers in bioethics, to find the words." Through the educational potential of her image-stories, she specifically wants "to use images to demonstrate how Canada's colonial history affects health outcomes of Indigenous people. I want this to be as common a practice as anatomy images are within medicine. I just want Indigenous people to feel safe going to a doctor. That's it."

However, when I ask her if she envisions her art as a form of social justice or advocacy, she is hesitant. The question leads her to reflect on two areas of ongoing development in her practice. First, she considers the impact on herself of being an advocate, and second, the limits of her knowledge. "You are right: I am an advocate," she tells me, "but I feel uncomfortable referring to myself as an advocate, because it burdens me with a tremendous responsibility." Part of that responsibility lies in recognizing that the knowledge she wishes to share with others is incomplete and is not hers alone to share. "I don't really feel knowledgeable," she says. "The knowledge is unfolding in the images. The knowledge is ancestral knowledge and is affirmed by land-based teaching that I received from my father before he died."

After our workshop in Sioux Lookout, Lisa shares how exposed the work makes her feel, and her fears that she is exposing her family, too. She wants to tell enough of her family's story to invoke change, but she is cautious not to reveal the details of the colonial indignities her family has suffered. "I have been asked incredibly inappropriate questions in public by members of audiences and workshop participants. Questions that would force me to go into detail. Questions about assumed abuses that I will not confirm or deny. These questions always lead to an uncomfortable silence, followed by a deep sigh and the pain of regret in my chest."

We reflect on this unavoidable voyeurism, the ethics of storytelling and the fact that you cannot control how a story is received. Through this conversation, I come to realize a foundation of Lisa's art, and perhaps of narrative in general: stories always exist relationally. And it is in the context of our relationship that the sharing of Lisa's art, the sharing of knowledge, the evolving sharing of bioethics, also unfold. Lisa agrees that "we have a relationship that is professional and personal, and in a relationship, both parties have to recognize the responsibilities of a relationship." She says, "Canada has failed in its relationships with Indigenous people." Perhaps this relational unfolding through viewing and witnessing Lisa's art offers the possibility of more responsible relationships between health care providers and Indigenous peoples.

\section{Allison Crawford MD PhD}

Centre for Addiction and Mental Health, Department of Psychiatry, University of Toronto, Toronto, Ont.

\section{References}

1. Charon R. Narrative medicine: honoring the stories of illness. Oxford (UK): Oxford University Press; 2008.

2. Mehl-Madrona L. Narrative medicine: the use of history and story in the healing process. Rochester (VT): Bear \& Co.; 2007.

3. Hooker C, Noonan E. Medical humanities as expressive of Western culture. Med Humanit 2011;37:79-84.

4. Berkhout S. Hearing between wor $(\mathrm{l}) \mathrm{ds}$ : rhetorical space and disrupting narratives in medicine. CMAJ 2017;189:E1494-6.

5. Bombay A, Matheson K, Anisman H. The intergenerational effects of Indian residential schools: implications for the concept of historical trauma. Transcult Psychiatry 2014;51:320-38.

6. Allan B, Smylie J. First Peoples, second class treatment: the role of racism in the health and well-being of Indigenous peoples in Canada. Toronto: Wellesley Institute; 2015.

7. Vizenor G. Fugitive poses: Native American Indian scenes of absence and presence. Lincoln (NE): University of Nebraska Press; 1998:15.

This article has been peer reviewed

Author's note: Conversations with Lisa Boivin occurred in person during this trip to Sioux Lookout, Oct. 25-27, 2017, and in a follow-up conversation in Toronto, on Dec. 1, 2017. Lisa vetted the contents of this article. She attributes the teaching "We are the land. The land is us" to the Dene artist Alex Janvier.

Editor's note: This is part one of two. Part two, which will be published September 17, features some of Lisa Boivin's images. 